\title{
Breathing pattern abnormalities in full term asphyxiated newborn infants
}

\author{
P Sasidharan
}

\begin{abstract}
Perinatal asphyxia is a cause of significant morbidity among full term infants, but breathing abnormalities after an asphyxic insult have not been studied. This report details breathing patterns of 16 full term asphyxiated infants, during the first week of life who were studied by transthoracic impedance pneumocardiograms. Pneumocardiograms were abnormal in $69 \%$ of infants in the asphyxiated group and $13 \%$ of infants in the control group. Significant differences were noted in the incidence of prolonged apnoea, the percentage of periodic breathing, and in apnoea density. These results indicate that there are significant abnormalities in the breathing pattern of full term infants, during their first week, after perinatal asphyxia. Similar abnormalities have been described in infants who had experienced 'near miss' sudden infant death syndrome.
\end{abstract}

Perinatal asphyxia is a cause of significant morbidity among full term infants. Among the central nervous system manifestations, hypoxicischaemic encephalopathy is the major event that leads to clinical symptoms including seizures. ${ }^{1}$ Many affected infants develop respiratory failure requiring artificial ventilation for a few days. Whether the respiratory failure is due to the direct effect of asphyxia on the respiratory center or to a global suppression of neuronal activity from severe hypoxic-ischaemic encephalopathy is not certain. We have observed several episodes of apnoea not associated with (electroencephalography proved) seizures in full term newborn infants during the postasphyxial state in the nursery. As the breathing pattern of full term neonates who suffered perinatal asphyxia has not been studied in the past, the objective was to study their breathing. Significant abnormalities in the breathing patterns of these full term asphyxiated newborns was found and the findings reported here. As similar abnormalities in breathing are seen in infants who had nearly experienced sudden infant death syndrome ('near miss' SIDS), hypoxia may possibly be the common initiating event responsible for such abnormalities.

Paediatrics,
Medical College
of Wisconsin,
Milwaukee, USA
Correspondence to:
Dr P Sasidharan,
Division of Neonatology,
Box 174, Milwaukee County
Medical Center,
8700 W Wisconsin Avenue,
Milwaukee, Wisconsin 53226,
USA.
Accepted 21 November 1991

Materials and methods

Sixteen full term infants of appropriate size for gestational age and with a history of perinatal asphyxia were studied. Asphyxia was defined as an Apgar score of 4 or less at 5 minutes after birth and an arterial $\mathrm{pH}$ of less than $7 \cdot 24$ with a base deficit in excess of -10 within 30 minutes after birth. Infants with clinical seizures were excluded from the study. Infants with congenital anomalies, congenital infections, and clinically proved early onset sepsis were excluded from the study. The mean (SD) birth weight was $3365(260) \mathrm{g}$ at a gestational age of 40 weeks (range $38-42$ weeks). There were 10 boys and six girls. Sixteen randomly selected full term neonates, also of appropriate size for gestational age, served as controls (eight boys and eight girls). Their mean Apgar score at 5 minutes was 9.4 (range 9-10), and their mean (SD) birth weight was 3310 (315) $\mathrm{g}$ at a gestational age of 39.9 weeks (range $38-42$ ).

Twelve hour nocturnal transthoracic impedance pneumocardiograms were obtained between 3 and 7 days of age. These recordings were made on a cassette tape which was subsequently analysed on a computer. The computer software is programmed to report the following variables: apnoea duration of greater than 15 seconds, of 11-15 seconds, or greater than 5 seconds; apnoea density; percentage of periodic breathing; bradycardia; and disorganised breathing.

Apnoea density $(\mathrm{A} 6 / \mathrm{D} \%)$ is defined as the total duration of apnoeic episodes of 6 seconds or longer divided by total sleep time and multiplied by 100 . Bradycardia is defined as heart rate of less than 80 per minute lasting 5 or more seconds. Periodic breathing is defined as three or more apnoeic spells of 3 seconds or longer interrupted by 20 seconds or less of normal breathing without apnoea; the total duration of periodic breathing is expressed as a percentage of total sleep time. Disorganised breathing is defined as short apnoeic episodes accompanied by bradycardia during sleep. Scoring of the pneumocardiogram was carried out by a computer equipped with software programmed to generate a report (Medical Graphics). ${ }^{2}$

The following criteria were used to define normal pneumocardiogram results. A pneumocardiogram is considered normal if there are no episodes of prolonged apnoea (apnoea lasting longer than 15 seconds in duration), apnoea density is less than 0.9 , and periodic breathing is less than $3.5 \%$ and there are no episodes of bradycardia nor disorganised breathing. These criteria have been published by others. ${ }^{3-7}$ All pneumocardiograms recorded on cassette tapes were analysed the next morning to generate hard copy at a recorder speed of $2 \cdot 4$ seconds $/ \mathrm{mm}$ of paper. Pneumocardiograms were done at a mean postnatal age of 4.06 days (range 3-6) in the control group and 5 days (range 4-7) in the asphyxiated group. Statistical analyses were carried out by two tailed $t$ tests for individual 
Breathing patterns of infants with asphyxia compared with those of controls

\begin{tabular}{llll}
\hline & Asphyxia & Control & $p$ Value \\
\hline Mean (SD) birth weight (g) & $3365(260)$ & $3310(315)$ & NS \\
No (\%) of infants with prolonged apnoea & $5(31)$ & 0 & $<0.05$ \\
Mean (SD) apnoea density & $1.41(0.87)$ & $0.59(0.26)$ & $<0.05$ \\
Mean (SD) \% periodic breathing & $3.12(0.8)$ & $2.13(0.67)$ & $<0.05$ \\
No (\%) with normal pneumogram & $5(31)$ & $14(88)$ & $<0.05$ \\
\hline
\end{tabular}

variables between the two groups and $\chi^{2}$ test with Yates's correction.

\section{Results}

Eleven infants $(69 \%)$ in the asphyxia group had abnormal pneumocardiogram results and five infants had normal results. Only two of the 16 infants in the control group had abnormal pneumocardiogram results $(p<0.05)$. Among infants with abnormal pneumocardiograms, five out of $11(45 \%)$ had prolonged apnoea $(>15 \mathrm{~s})$; nine out of $11(82 \%)$ had increased apnoea density and six out of $11(55 \%)$ had abnormal periodic breathing. The two abnormal pneumocardiograms in the control group were due to increased apnoea density, with other parameters being normal. We did not analyse the episodes of apnoea 11-15 seconds' duration and episodes of periodic breathing per 100 minutes of sleep time. There were no episodes of isolated bradycardia or disorganised breathing in either group. The abnormalities in the breathing pattern seen among the asphyxiated group were significant $(p<0.05)$ when compared with the control group in the following variables: prolonged apnoea, percentage of periodic breathing, and apnoea density (see table).

\section{Discussion}

Breathing patterns of full term asphyxiated newborn infants have not been studied in the past. Our results indicate that the breathing patterns are abnormal among asphyxiated infants during the first week of life, but whether these changes are induced by the asphyxia is not known. It had been reported that apnoea in newborn infants is associated with a history of antecedent hypoxia. ${ }^{8}$ Infants who had near miss SIDS were reported to have abnormal breathing patterns identified by pneumograms. 34 9-11 The abnormalities described in those infants are similar to those which we detected in our group of asphyxiated neonates. There is no information on the breathing pattern of these infants preceding an asphyxial insult. If an hypoxic insult to the brain produced these abnormalities in breathing patterns, then the abnormalities reported in the past in these near miss SIDS infants might also be due to the hypoxic events. If so, then the assessment of breathing patterns should have no predictive value for SIDS, as the hypoxia may be an acute event immediately preceding death. However, the assessment of breathing patterns offers an added tool in evaluating the severity of (or effect of) asphyxia on central respiratory centre.

Pneumocardiograms have been used as a tool in assessing breathing patterns in infants with apnoea of prematurity, apnoea of infancy, near miss SIDS, infants on home apnoea monitors, siblings of SIDS infants, and those born to mothers practising substance abuse during pregnancy. ${ }^{3} 1012-14$ The predictive value of pneumocardiograms in SIDS is very controversial. $^{15-17}$ Several variables in the pneumocardiograms can be analysed. We chose to use prolonged apnoea, percentage of periodic breathing, apnoea density, bradycardia, and disorganised breathing as the variables in the study of breathing pattern. All pneumocardiograms in this study were recorded in the hospital. As computer aided pneumogram scoring and interpretation reduce the interpersonal bias involved in the reports, we employed this technique. ${ }^{2} 18$

None of our non-asphyxiated (control group) infants had prolonged apnoea. This is similar to the reported findings of Kelly et al. ${ }^{4}$ The increased apnoea density noted in two infants in the control group was due to multiple episodes of apnoea of 6-14 seconds' duration. None of the infants in either group had obstructive apnoea. The reliability of detection of obstructive apnoea from pneumocardiogram tracings is debatable. Bradycardia is a usual accompaniment of obstructive apnoea and as we could not detect any episodes of bradycardia with chest wall movement, we made the assumption that there were no obstructive apnoea spells. All our infants were breathing spontaneously in room air when we studied them. There were no episodes of cyanosis in any of our infants. We did not monitor the infants with an oximeter to record oxygen saturation continuously during the study.

It is known that preterm infants increased periodicity in the breathing pattern and this is abolished by oxygen administration. ${ }^{19}$ If periodic breathing and subsequent apnoea are due to mild hypoxia in preterm infants, the effects of acute hypoxia and acidosis as seen in perinatal asphyxia may last several days. It is possible that the observed changes in the breathing pattern of these infants may be due to that; but our infants were full term and such changes have not been described in the past. In conclusion, we have identified abnormalities in the breathing pattern of full term asphyxiated newborn infants during the first week of life. The duration of the persistence of these abnormalities in the breathing pattern needs further studies in future.

We greatly appreciate and sincerely thank Professor RDG Milner for reviewing this manuscript and for the helpful suggestions and criticisms.

This paper was presented in part at the Third International Conference on Fetal and Neonatal Physiological Measurements, Ronneby, Sweden, June 1988.

1 Volpe JJ. Neurology of the newborn. Philadelphia: WB Saunders, 1981.

2 Sasidharan P, Marquez E, Dizon E, Sridhar CV. Comparative analysis of pneumogram scoring by a computer assisted system vs manual scoring. Pediatr Res 1985;19:415A.

3 Kelly DH, Shannon DC. Periodic breathing in infants with near miss sudden infant death syndrome. Pediatrics 1979 63:355-60.

4 Kelly DH, Stellwagen LM, Kaitz E, Shannon DC. Apnea and periodic breathing in normal full term infants during the first twelve months. Pediatr Pulmonol 1985;1:215-9. the first twelve months. Pediatr Pulmonol 1985;1:215-9. Weissblunt Brouillette RT, Hanson D, David RJ, Stein IM J Pediatr 1985; 106:551-5. 
6 Hunt CE, Brouillette RT, Liu Klemka L. Day-to-day pneumogram variability. Pediatr Res 1985;19:174-7. 7 Steinschneider A. Prolonged sleep apnea and respiratory 962-70.

8 Naeye RL. Neonatal apnea: underlying disorders. Pediatrics 1979;63:8-12.

9 Oren J, Kelly DH, Shanon DC. Pneumogram recordings in infants resuscitated for apnea of infancy. Pediatrics 1989; 83:364-8.

10 Kelly DH, Golub H, Carley D, Shannon DC. Pneumograms in infants who subsequently died of sudden infant death syndrome. F Pediatr 1986;109:249-54.

11 Oren J, Kelly DH, Shannon DC. Familial occurrence of sudden infant death syndrome and apnea of infancy. Pediatrics 1987;80:355-8.

12 Flores-Guevara R, Sternberg B, Peirano P, Guidasci S, Durupt N, Monod N. Respiratory pauses and periodic breathing assessed by cardio-pneumography in normal infants and in SIDS siblings. Neuropediatrics 1986;17: 59-62.

13 Anwar M, Mondestin H, Mojica N, et al. Effect of caffeine on pneumogram and apnoea of infancy. Arch Dis Child 1986;61:891-5.

14 Hunt CE, Brouillette RT, Hanson D. Theophylline improves 作 F Pediatr 1983;103:969-74.

15 Southall DP, Richards JM, Rhoden KJ, et al. Prolonged apnea and cardiac arrhythmias in infants discharged from apnea and insive care units: failure to predict an increased risk for sudden infant death syndrome. Pediatrics 1982;70: 844-51.

16 Southall DP, Richards JM, deSwiet M, et al. Identification of infants destined to die unexpectedly during infancy: evaluation of predictive importance of prolonged apnea and disorders of cardiac rhythm or conduction. $B M \mathcal{J} 1983 ; 286$ : disorders

17 Monod N, Plouin P, Sternberg B, et al. Are polygraphic and cardiopneumographic respiratory patterns useful tools for predicting the risk of sudden infant death syndrome? Biol Neonate 1986;50:147-53.

18 Golub H, Kelly DH, Shannon DC. Computer analysis of pneumograms: abstracted. Pediatr Res 1984;17:373A.

19 Rigatto H. Apnea and periodic breathing. Semin Perinatol 1977;1:375-81. 